\section{Kinetics of HIV-1 in cerebrospinal fluid and plasma in cryptococcal meningitis}

\author{
Diego M. Cecchini, ${ }^{1}$ Ana M. Cañizal, ${ }^{2}$ \\ Haroldo Rojas, ${ }^{1}$ Alicia Arechavala, ${ }^{3}$ \\ Ricardo Negroni, ${ }^{3}$ María B. Bouzas, ${ }^{2}$ \\ Jorge A. Benetucci ${ }^{1}$ \\ 'Infectious Diseases Department, \\ ${ }^{2}$ Virology Unit and ${ }^{3}$ Mycology Unit, \\ Hospital Francisco J. Muñiz, Buenos \\ Aires, Argentina
}

\section{Abstract}

In order to determine HIV-1 kinetics in cerebrospinal fluid (CSF) and plasma in patients with cryptococcal meningitis (CM), we undertook a prospective collection of paired CSF/plasma samples from antiretroviral therapy-free HIV-infected patients with $\mathrm{CM}$. Samples were obtained at baseline (S1) and at the second (S2) and third (S3) weeks of antifungal therapy. HIV-1 CSF concentrations were significantly lower in both S2 and S3 with respect to $\mathrm{S} 1$. Plasma concentrations remained stable. HIV-1 concentrations were higher in plasma than CSF in all cases. Patients who survived the episode of $\mathrm{CM}$ (but not those who died) showed a decrease in CSF viral load, what suggests different viral kinetics of HIV-1 in the CSF according to the clinical course of this opportunistic disease.

\section{Introduction}

The HIV-1 retrovirus is found in the cerebrospinal fluid (CSF) of most HIV-infected individuals at all stages of HIV infection, including primary infection and the asymptomatic and symptomatic phases. The centra nervous system (CNS) is considered a sanctuary site for the replication of HIV-1, as it acts as an anatomical structure in which the virus can replicate and evolve independently of viral particles found in other organs. ${ }^{1,2}$

CNS opportunistic infections are a major cause of morbidity and mortality in HIV-1 infected subjects. ${ }^{3}$ However, little is known about HIV-1 dynamics in the context of such diseases. Cross-sectional studies have investigated the concentrations of this retrovirus in the CSF of patients with various opportunistic neurological diseases but without prospective monitoring of viral load values. ${ }^{4-7}$ Therefore, whether the treatment of an opportunistic neurological infection modifies concentrations of HIV-1 in the CSF is unknown.
Investigations of HIV-1 viral load in the CSF have included limited numbers of patients with CNS opportunistic infections. For example, only a small number of patients with cryptococcal meningitis have been studied. ${ }^{4-8}$ Yet, cryptococcal meningitis is an appropriate model for studying HIV-1 kinetics in the CSF because microbiologic diagnosis can be done easily (via the Indian ink test), lumbar puncture is mandatory for routine diagnosis and follow-up of the disease (particularly if it evolves with high intracranial pressure), and standardized practice guidelines exist. ${ }^{3}$

\section{Brief Report}

We previously reported on factors that influence CSF HIV-1 concentrations in patients with meningitis by Cryptococcus neoformans. ${ }^{9}$ The purpose of the present investigation was to study HIV-1 dynamics during the first 3 weeks of antifungal therapy and to compare concentrations of HIV-1 in the CSF and in the plasma of patients with cryptococcal meningitis. We conducted a longitudinal observational study at a single center. The study took place at the Francisco J. Muñiz Infectious Diseases Hospital in Buenos Aires, Argentina, from 2004 to 2006. We prospectively collected and analyzed paired CSF and blood samples from HIVpositive patients with culture-confirmed cryptococcal meningitis. Because of the observational nature of the study, all CSF samples were collected on the primary physician's judgment as part of routine care for patients with cryptococcal meningitis based on local/institutional standards and international recommendations. All procedures were in accordance with the institution's ethical standards and with the Helsinki Declaration of 1975 , as revised in 1983. Informed consent was obtained in all cases.

All samples were routinely cultured on Sabouraud agar plates to enable the detection of fungal pathogens and in other media to enable the detection of mycobacteria (LowensteinJensen) and common aerobic bacteria (blood agar). Patients with cultures that tested positive for pathogens other than Cryptococcus neoformans or who showed evidence of another simultaneous CNS infection were excluded from the sample. None of the patients received antiretroviral drugs before or during the study. For each patient, we measured CSF HIV and plasma viral loads simultaneously (in the same assay) by polymerase chain reaction using the Cobas Amplicor Monitor Test version 1.5 (Roche Diagnostic Systems, Inc., Branchburg, NJ) and following the manufacturer's instructions. CSF samples (with a paired plasma sample) were obtained at the moment of the diagnosis of cryptococcal meningitis and prior to the onset of
Correspondence: Diego M. Cecchini, Hospital Cosme Argerich, Pi y Margall 750, Buenos Aires 1155, Argentina.

Tel. +5411.41210828 - Fax: +5411.4307 5952 .

E-mail: diegocec@gmail.com

Key words: meningitis, HIV, Cryptococcus neoformans, cerebrospinal fluid.

Acknowledgments: the authors would like to thank María del Carmen Iannella, University of Buenos Aires, for the statistical support.

Contributions: DMC, design of the study, patient enrollment, data analysis, and writing of the manuscript; AMC, proceedings performed in the Virology Unit, and was co-writer of the manuscript; HR, patient enrollment; AA, RN, proceedings performed in the Mycology Unit; MBB, design of the study, was supervisor of the proceedings performed in the Virology Unit, and cowriter and final supervisor of the manuscript; $\mathrm{JAB}$, design and general supervision of the study, and was co-writer and final supervisor of the manuscript.

Conflict of interests: the authors report no conflict of interests.

Received for publication: 11 November 2011. Revision received: 23 March 2012.

Accepted for publication: 4 April 2012.

This work is licensed under a Creative Commons Attribution NonCommercial 3.0 License (CC BYNC 3.0).

(C) Copyright D.M. Cecchini et al., 2012 Licensee PAGEPress, Italy

Infectious Disease Reports 2012; 4:e30 doi:10.4081/idr.2012.e30

antifungal therapy [sample 1 (S1)] and at the second [sample 2 (S2)] and third [sample 3 (S3)] weeks of treatment with antifungal therapy. Data were processed using the Wilcoxon rank sum test and Wilcoxon signed rank test for the analysis of nonparametric quantitative data (Statistix 7.0; Analytical Software, Tallahassee, $\mathrm{FL})$. CSF and plasma S1 were compared to S2 and S3 for all patients. S1 and S3 were compared between patients who survived the episode of cryptococcal meningitis and those who died. In addition, a last observation carried forward (LOCF) approach for CSF measurements was done: in this analysis we assign the same HIV RNA value at S2 and S3 that was measured at $\mathrm{S} 1$ if the patient died between $\mathrm{S} 1$ and S2; or, similarly, the same value at S3 that was measured at $\mathrm{S} 2$ if the patient died between S2 and S3. CSF and blood samples from 37 HIVinfected patients with culture-confirmed cryptococcal meningitis were collected prior to antifugal treatment. CSF samples from 3 patients were excluded because of the diagnosis of a simultaneous CNS opportunistic disease 
(either Cytomegalovirus encephalitis, cerebral toxoplasmosis, or Chagas's encephalitis). Therefore, 34 patients met the inclusion criteria for S1. Of these, 10 had an S2 and 29 had an S3. Patients were 35 years old (32-42), 74\% of them were male, and the $\mathrm{CD} 4^{+} \mathrm{T}$ lymphocyte count was 24 cells $/ \mathrm{mm}^{3}$ (11-43; all data presented as the median, interquartile range). The CSF cytological and biochemical characteristics were as follows: leukocyte count, 10 cells $/ \mathrm{mm}^{3}$ (4-23); glucose, $39 \mathrm{mg} / \mathrm{dL}$ (32.7-50); protein concentration $0.75 \mathrm{~g} / \mathrm{L}$ (0.48-1.06); and Cryptococcus neoformans capsular polysaccharide antigen titer, 1/100 dilutions (1/10-1/1000). ${ }^{9}$ Eighty-five percent of patients had a positive CSF India ink examination. No difference was observed between survivors and non survivors in the CSF cellularity $(\mathrm{P}=0.31)$, protein concentration $(\mathrm{P}=0.81)$ and Cryptococcus neoformans capsular polysaccharide antigen titer $(\mathrm{P}=0.27)$. CSF glucose concentration was significantly lower in nonsurvivors [27 (17-46) mg/dL] vs. survivors [40 (38-75) mg/dL], $P=0.03$. All patients received antifungal treatment after diagnosis; amphotericin B monotherapy was the most common regimen (32 patients, 94.1\%). A total of 18 patients (54.4\%) evolved with high intracranial pressure; 23 patients (67.6\%) survived. Mortality was higher among patients with high opening pressures at baseline (Odds ratio: $6.5,95 \%$ confidence interval: $1.12-37.4, \mathrm{P}=0.06)$. Plasma HIV-1 median viral load values (expressed as $\log 10$ copies $/ \mathrm{mL}$ ) were 3 to $>5.88$ (S1), 3.12 to $>5.88$ (S2), and 4.04 to $>5.88$ (S3). The three samples did not differ significantly in terms of HIV-1 concentration ( $\mathrm{P}>0.05)$.

CSF HIV-1 viral load values were $4.83(<2.6$ to $>5.88 ; \mathrm{S} 1), 3.53$ ( $<2.6$ to $4.99 ; \mathrm{S} 2)$, and 4.33 ( $<2.6$ to $5.61 ; \mathrm{S} 3$ ). The S2 and S3 values did not differ significantly $(\mathrm{P}=0.94)$, but both were significantly lower than the $\mathrm{S} 1$ value $(\mathrm{P}=0.04$ and $\mathrm{P}=0.02$, respectively). Results remained unchanged with LOCF approach. The evolution of CSF HIV concentrations from baseline to the second week of antifungal therapy is shown in Figure 1. HIV-1 concentrations were significantly higher in the plasma than in the CSF for S1, S2, and S3 ( $\mathrm{P}=0.001, \mathrm{P}=0.008$ and $\mathrm{P}<0.0001$, respectively). When we stratified the CSF viral loads according to the overall clinical outcome, we found that $\mathrm{S} 3$ values were significantly lower than $\mathrm{S} 1$ values $(\mathrm{P}=0.03)$ for survivors, as follows: $4.79(<2.6$ to $>5.88, \mathrm{n}=$ 23 ; 1$)$ and 4.21 ( $<2.6$ to $5.39, \mathrm{n}=20 ; \mathrm{S} 3)$. The S3 CSF viral load did not differ from the S1 viral load ( $\mathrm{P}>0.05)$ for nonsurvivors: 4.87 $(<2.6$ to $>5.88, \mathrm{n}=11 ; \mathrm{S} 1)$ and $4.61(<2.6$ to $5.61, \mathrm{n}=9 ; \mathrm{S} 3)$. Results remained unchanged with LOCF approach (Figure 2). No difference was observed between S1 and S3 ( $>>0.05)$ plasma samples for either survivors or nonsurvivors (data not shown).

\section{Discussion}

Our study suggests that in a population of patients with culture-confirmed cryptococcal meningitis, HIV-1 has different dynamics in the CSF and plasma: CSF concentrations decreased at 2 weeks of antifungal therapy, whereas plasma concentrations remained stable. This result further supports previous find- ings, including ours, that suggest the existence of independent viral kinetics in plasma and $\mathrm{CSF}^{8-10}$

Determining the pathophysiologic mechanism of such independent viral kinetics is beyond the scope of this study, and thus we can only hypothesize here. One possibility might be that treatment of meningeal cryptococcosis reduces the concentration of proinflammatory cytokines in the CSF. These cytokines stimulate

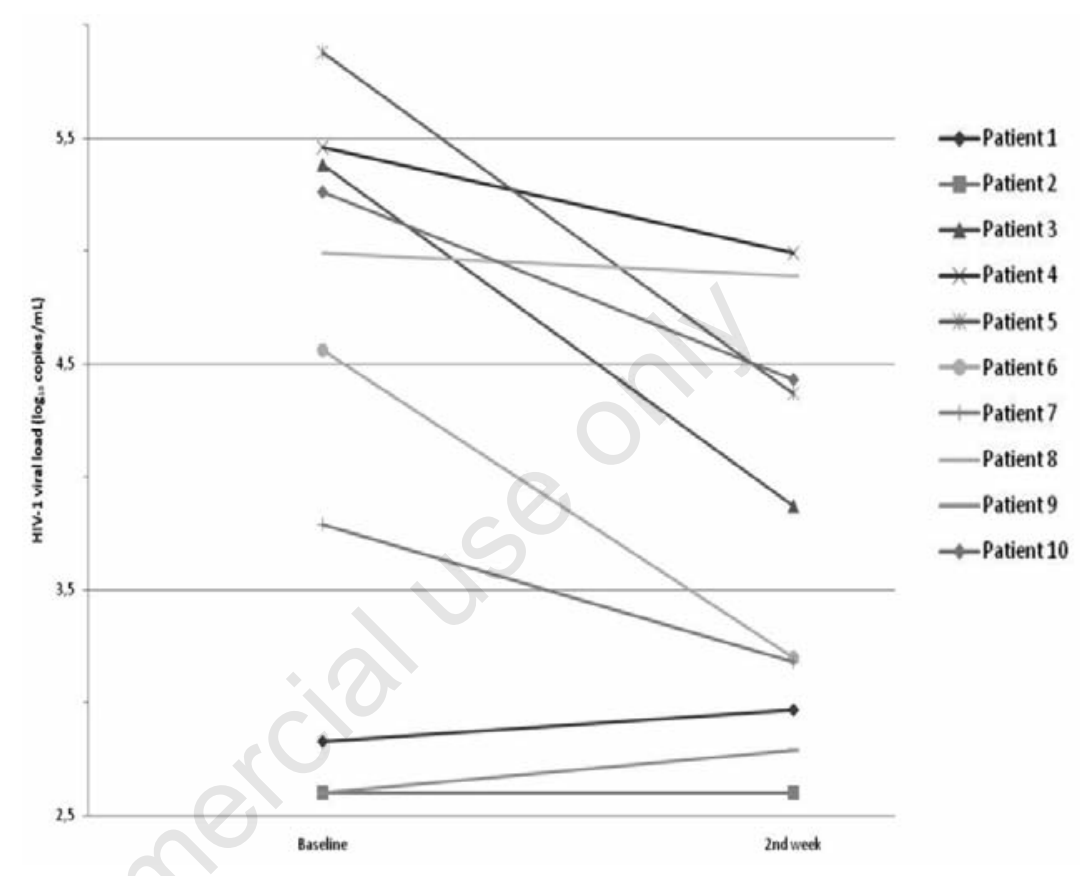

Figure 1. Evolution of cerebrospinal fluid HIV-1 viral load in patients with cryptococcal meningitis from baseline to the second week of antifungal therapy.

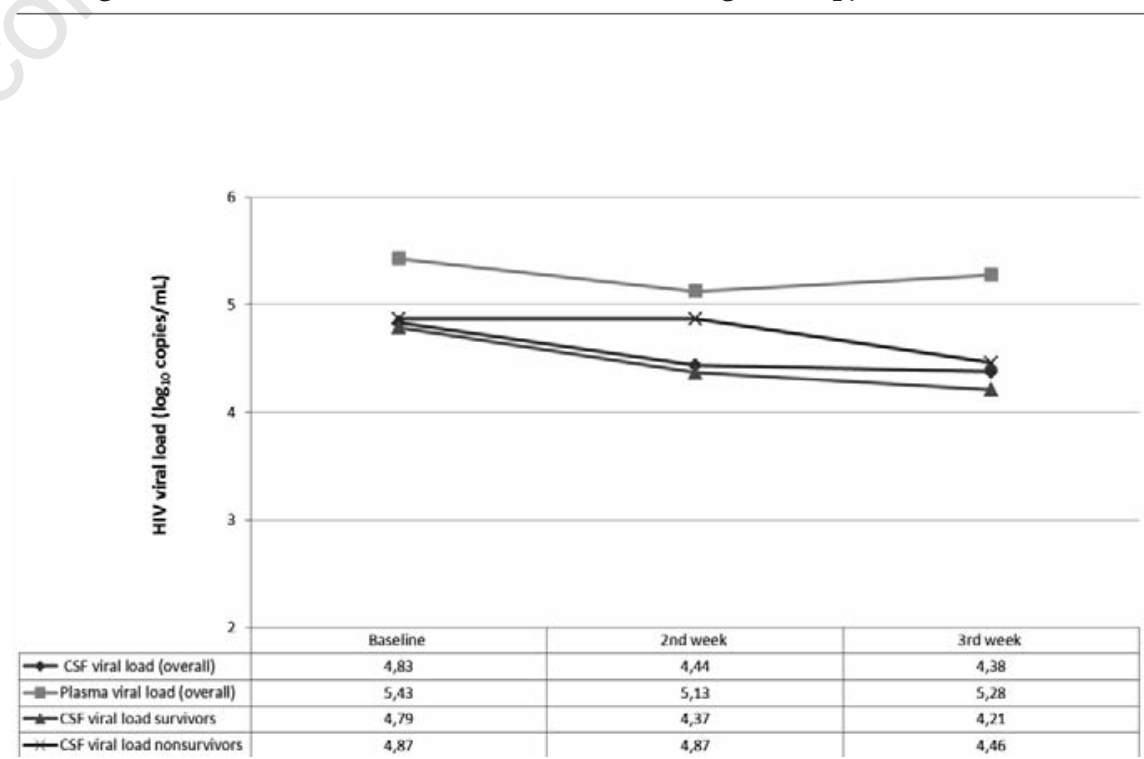

Figure 2. Three-week evolution of HIV-1 viral loads (median values) in the cerebrospinal fluid and plasma of 34 patients with cryptococcal meningitis. CSF viral load evolution is shown for the overall population and stratified by final outcome (last observation carried forward approach, see text). 
the recruitment of inflammatory cells into the CNS and favor the activation of lymphocytes, monocytes, and local resident cells infected by HIV-1, which stimulates viral replication and increases the viral load in the CSF. ${ }^{11-13}$ With the reduction in the fungal burden in the CNS as a result of antifungal therapy, the concentrations of these cytokines as well as proinflammatory phenomena decrease, reducing viral replication. Previous research has shown that concentrations of cytokines such as IL-6, IFN-gamma, GM-CSF, IL-8, TNF-alpha, and macrophage inflammatory protein-1 (MIP-1) in the CSF of patients with cryptococcal meningitis are higher at baseline (before the onset of antifungal therapy) than at 7 and 14 days of treatment. Of note is that CSF concentrations of these cytokines are independent of the cellularity of the fluid. ${ }^{11}$ This suggests a decline in local immune stimulation to the extent that the infection is controlled. A recent investigation characterized the statistical association between serum cytokine profiles and immune reconstitution inflammatory syndrome in cryptococcal meningitis, ${ }^{14}$ but information is still limited regarding the CSF cytokine profile and its impact on HIV-1 concentrations, the evolution of CNS inflammatory phenomena, and clinical outcomes. Considering these data, the influence of cytokines in the kinetics of HIV-1 in the CNS should be further investigated. Another original aspect of this study is the preliminary observation of different viral dynamics observed in the CSF according to the final clinical outcome. In our cohort, patients who survived the episode of meningitis showed a decrease in HIV-1 concentrations at the third week of treatment compared to baseline. In contrast, patients who died had stable CSF values. This finding was not replicated in the plasma, where viral load values remained stable regardless of the final clinical outcome. The present series is, to the best of our knowledge, the first to suggest different viral kinetics in the CSF according to the clinical course of this opportunistic disease. However, as few subjects died during this study, this preliminary finding should be confirmed with a larger number of patients in order to turn this observation a firm conclusion. Furthermore, stratification according to baseline severity of cryptococcal infection is required in future cohorts in order to determine an association (if any) between HIV CSF kinetics and the final clinical outcome of this opportunistic infection. This is of major importance, as if HIV kinetics in the CSF negatively impact the outcome of central nervous system co-infections, this may have clinical implications in terms of prompt initiation of antiretroviral therapy or early assessment of CSF viral reservoir when such infections are diagnosed.
This study has several additional limitations. First, the sampling of patients in this cohort occurred over 3 weeks, the usual length of time for the induction phase of treatment of cryptococcosis in our setting. The study design did not include sampling of CSF and plasma beyond that period, as most of the patients had started the highly active antiretroviral therapy, which significantly reduces the viral load in both the plasma and CSF. ${ }^{15}$ Also, we did not investigate HIV-1 concentrations in the CSF and plasma during the first week of treatment of cryptococcosis. This is a major limitation, as this is the period with the highest mortality, for which our findings may have a clinical implication (i.e., early viral dynamics as a prognostic factor in cryptococcal meningitis).

Second, the changes found in viral load in the CSF were modest. However, greater differences would have been unlikely as patients were not exposed to antiretroviral therapy and our population had severely compromised immunity, which is usually associated with high viraemia. Third, we were unable to measure cytokines in the CSF and plasma. As previously stated, analysis of these molecules in the CSF would have provided important information, as their influence on HIV-1 concentrations in the CSF remains unknown. However, measurement of cytokines in CSF is not routinely done in our institution and resources could not be allocated for the purpose of this study. Finally, our results are representative of patients with meningitis caused by Cryptococcus neoformans only and cannot be extrapolated to patients with other CNS infections, such as Cytomegalovirus encephalitis, tuberculous meningitis, progressive multifocal leukoencephalopathy, or cerebral toxoplasmosis. The observational design of this study (chosen because of ethical constraints regarding the risks of the lumbar puncture procedure) precluded the inclusion of a control group of asymptomatic subjects (i.e., those without cryptococcal meningitis).

\section{Conclusions}

In conclusion, this investigation is, as far as we know, the first longitudinal study to assess the kinetics of HIV-1 in the CSF of patients with a specific CNS infection and without antiretroviral therapy. Our results suggest independent dynamics in the CSF and plasma. Further investigations are needed to elucidate the clinical implications of HIV-1 kinetics in the CSF in the context of cryptococcal meningitis and other CNS opportunistic diseases.

\section{References}

1. Fierer DS, Klotman ME. Kidney and central nervous system as reservoirs of HIV infection. Curr Opin HIV AIDS 2006;1: 115-20.

2 Garcia F, Niebla G, Romeu J, et al. Cerebrospinal fluid HIV-1 RNA levels in asymptomatic patients with early stage chronic HIV-1 infection: support for the hypothesis of local virus replication. AIDS 1999;13:1491-6.

3. Perfect JR, Dismukes WE, Dromer F, et al. Clinical practice guidelines for the management of cryptococcal disease: 2010 update by the Infectious Diseases Society of America. Clin Infect Dis 2010;50:291322 .

4. Morris L, Silber E, Sonnenberg P, et al. High human immunodeficiency virus type 1 RNA load in the cerebrospinal fluid from patients with lymphocytic meningitis. J Infect Dis 1998;177:473-6.

5. Claes M, Albert J, Hansson P, et al. Cerebrospinal fluid mononuclear cell counts influence CSF HIV-1 RNA levels. J Acquir Immune Defic Syndr Hum Retrovirol 1998;17:214-9.

6. Brew BJ, Pemberton L, Cunningham P, Law MG. Levels of human immunodeficiency virus type 1 RNA in cerebrospinal fluid correlate with AIDS dementia stage. J Infect Dis 1997;175:963-6.

7. Christo P, Greco D, Aleixo A, Livramento J. HIV-1 RNA levels in cerebrospinal fluid and plasma and their correlation with opportunistic neurological diseases in a Brazilian AIDS reference hospital. Arq Neuropsiquiatr 2005;63:907-13.

8. Spudich SS, Nilsson AC, Lollo ND, et al. Cerebrospinal fluid HIV infection and pleocytosis: relation to systemic infection and antiretroviral treatment. BMC Infect Dis 2005;5:98.

9. Cecchini DM, Cañizal AM, Rojas $\mathrm{H}$, et al. Variables that influence HIV-1 cerebrospinal fluid viral load in cryptococcal meningitis: a linear regression analysis. J Int AIDS Soc 2009;2:8.

10. Ellis RJ, Gamst AC, Capparelli E, et al. Cerebrospinal fluid HIV RNA originates from both local CNS and systemic sources. Neurology 2000;54:927-36.

11. Siddiqui AA, Brouwer AE, Wuthiekanun V, et al. IFN gamma at the site of infection determines the rate of clearance of infection in cryptococcal meningitis. J Immunol 2005;174:1746-0.

12. Chaka W, Heyderman R, Gangaidzo I, et al. Cytokine profiles in cerebrospinal fluid of human immunodeficiency virus- 
infected patients with cryptococcal meningitis: no leukocytosis despite high interleukin-8 levels. J Infect Dis 1997; 176:1633-6.

13. Lortholary 0, Dromer F, MathoulinPélissier S, et al. Immune mediators in cerebrospinal fluid during cryptococcosis are influenced by meningeal involvement and human immunodeficiency virus serostatus. J Infect Dis 2001;183:294-302.

14. Boulware DR, Meya DB, Bergemann TL, et al. Clinical features and serum biomarkers in HIV immune reconstitution inflammatory syndrome after cryptococcal meningitis: a prospective cohort study. PLoS Med 2010;7:e1000384.
15. Yilmaz A, Fuchs D, Hagberg L, et al. Cerebrospinal fluid HIV-1 RNA, intrathecal immunoactivation, and drug concentrations after treatment with a combination of saquinavir, nelfinavir, and two nucleoside analogues: the M61022 study. BMC Infect Dis 2006;6:63. 